\title{
Fully Differential Rates for Femtosecond Multiphoton Double Ionization of Neon
}

\author{
M. Weckenbrock, ${ }^{1}$ D. Zeidler, ${ }^{2}$ A. Staudte, ${ }^{1}$ Th. Weber, ${ }^{1}$ M. Schöffler, ${ }^{1}$ M. Meckel, ${ }^{1}$ S. Kammer, ${ }^{1}$ M. Smolarski, ${ }^{1}$ \\ O. Jagutzki, ${ }^{1}$ V. R. Bhardwaj, ${ }^{2}$ D. M. Rayner, ${ }^{2}$ D. M. Villeneuve, ${ }^{2}$ P. B. Corkum, ${ }^{2}$ and R. Dörner ${ }^{1, *}$ \\ ${ }^{1}$ Institut für Kernphysik, Universität Frankfurt, D-60486 Frankfurt, Germany \\ ${ }^{2}$ National Research Council, 100 Sussex Drive, Ottawa, Ontario, Canada K1A OR6
}

(Received 2 February 2004; published 28 May 2004)

\begin{abstract}
We have investigated the full three-dimensional momentum correlation between the electrons emitted from strong field double ionization of neon when the recollision energy of the first electron is on the order of the ionization potential. The momentum correlation in the direction perpendicular to the laser field depends on the time difference of the two electrons leaving the ion. Our results are consistent with double ionization proceeding through transient double excited states that field ionize.
\end{abstract}

DOI: $10.1103 /$ PhysRevLett.92.213002

Ejection of two electrons from an atom by absorption of one or more photons is key to understanding few-body dynamics. In the single photon limit, experiments accurately measure fully differential cross sections from $100 \mathrm{meV}$ [1] to $450 \mathrm{eV} \mathrm{[2]} \mathrm{above} \mathrm{threshold} \mathrm{[3].} \mathrm{Theory}$ is also highly developed and reproduces the experimental data very well [4-7].

For the case of multiphoton absorption in ultrashort Ti:Sa laser pulses $\left(\approx 100 \mathrm{fs}, 10^{14} \mathrm{~W} / \mathrm{cm}^{2}\right.$ range $)$, the situation is much less favorable. Experiments have measured the total double ionization rates [8], ion momenta $[9,10]$, single electron energy [11-13], and angular distributions [13], as well as the correlation between the momentum components either parallel $[14,15]$ or perpendicular [16] to the laser field. Major features, like the double hump structure in the ion momenta and the correlation in the electron momenta parallel to the field, have been reproduced by quantum and classical calculations [17].

Experiments do not yet measure fully differential cross sections, and it is difficult for theory to make reliable predictions of differential cross sections [18,19]. A full characterization of atomic double ionization requires measurement of the three momentum components of both electrons or of one of the electrons and the ion. All experiments reported so far have left at least one momentum component unobserved.

We report an experiment where all kinematical observables, i.e., the three-dimensional momentum vectors of both electrons, are determined. This allows us to relate the parallel and perpendicular momentum correlation and obtain fully differential angular distributions.

The physical mechanism responsible for these primary features is double ionization via rescattering [20,21]: The first electron is set free by the field, accumulates energy during about half a laser cycle, and is driven back to its parent ion where it can reemit a second electron. This may happen either via a direct or laser-assisted [22] electron impact ionization [21] or via the creation of intermediate excited ionic states which is later field ionized [15].
PACS numbers: $32.80 . \mathrm{Rm}, 31.90 .+\mathrm{s}, 32.80 . \mathrm{Fb}, 32.80 . \mathrm{Wr}$

For recollision electrons near threshold, we find that the electrons have little momentum in the direction perpendicular to the laser field - about the same as for single ionization in the same laser field (for example, argon [23]). We also find that the two electrons often have similar momentum in the direction parallel to the laser field. Assuming that this momentum is primarily obtained from the laser field, we deduce that both electrons are reemitted at about $30^{\circ}$ before or after the maximum of the laser field. Electrons that have similar longitudinal velocity repel each other in the lateral direction, confirming that they leave that atom at about the same time.

These observations are consistent with the recollision electron forming doubly excited states. These states live for a fraction of a laser cycle and then ionize when the field nears its maximum value [24,25].

The experiment was performed using cold target recoil ion momentum spectroscopy [26]. Ti:sapphire laser pulses (40 fs, $800 \mathrm{~nm}, 3.9 \mu \mathrm{J}, 30 \mathrm{kHz}$ repetition rate) are focused to peak intensity of $(1.9 \pm 0.3) \times 10^{14} \mathrm{~W} / \mathrm{cm}^{2}$ into a supersonic neon gas jet.

Ions and electrons created in the focus are guided by a $3.9 \mathrm{~V} / \mathrm{cm}$ electric and a $10.8 \mathrm{G}$ magnetic field towards two large area channel plate detectors with delay-line position encoding (www.Roentdek.com). The internal temperature of the gas jet, which determines the momentum resolution for the ion momentum measurement, is less perpendicular than parallel to its propagation direction. We chose the laser polarization parallel to the gas jet, ensuring that we have the highest momentum resolution perpendicular to the laser polarization. The momentum resolution was monitored simultaneously in the single ionization channel where ion and electron momenta have to compensate. We achieve a momentum resolution of \pm 0.035 a.u. along and \pm 0.15 and \pm 0.325 a.u. perpendicular to the spectrometer axis. The last value gives the resolution along the jet direction and is determined by the jet temperature. The settings of the electric and magnetic fields yielded a $4 \pi$ solid angle for electrons up to $35 \mathrm{eV}$. 
The momentum of the unobserved electron was calculated by taking the difference of the momentum of the observed and that of the ion.

The electron count rate was 0.1 per shot and the ion rate 0.02 . For single ionization, real coincidences are identified by momentum conservation between ion and electron (see Fig. 1 in [14]). The fraction of false coincidences in which the registered ion and electron result from two different atoms, both ionized in the same shot, was monitored in the single ionization channel to be $9 \%$.

A laser intensity of $1.9 \times 10^{14} \mathrm{~W} / \mathrm{cm}^{2}$ corresponds to a ponderomotive energy $U_{p}=11.3 \pm 2 \mathrm{eV}$. The usual value for the maximum return energy of the rescattered electron is $3.17 U_{p}=36 \pm 6 \mathrm{eV}$. However, for low intensities, the electron becomes classically free at a significant distance from the ion. The electron acquires $\sim 5 \mathrm{eV}$ added kinetic energy as a result of the added distance the electron travels in the laser field [27]. The total is $\sim 41 \pm$ $6 \mathrm{eV}$. This has to be compared to the field-free ionization potential of $41 \mathrm{eV}$ for the $\mathrm{Ne}^{+}$ion and the lowest excitation energy of $27 \mathrm{eV}$ for the first excited state of $\mathrm{Ne}^{+}$.

Since the recollision energy is close to threshold for nonsequential double ionization, we must consider the influence of the time dependent field on the instantaneous ionization potential [28] of the parent ion. Following the arguments in [28], we assume a cosine electric field $E(t)=E_{o} \cos \omega t$, where we neglect the time dependence of the pulse envelope $E_{o}$. The superposition of the laser electric field and a simplified hydrogenlike binding potential is given in cylindrical coordinates $(z, r, \theta)$ by

$$
V(z, r, \theta, t)=-\frac{Z}{\sqrt{z^{2}+r^{2}}}-E(t) z
$$

with $Z$ being the charge state of the ion. (Atomic units are used in this and all other equations.) This shows that the ionization barrier depends on space and time. Twice per laser cycle the barrier minimizes along the laser polarization direction $(r=0)$. An electron that is guided through this barrier by the field must be radially confined. For $Z=2$ and a field strength of $3.8 \times 10^{10} \mathrm{~V} / \mathrm{m}$ (corresponding to the maximum field of a $1.9 \times 10^{14} \mathrm{~W} / \mathrm{cm}^{2}$ pulse), the instantaneous ionization potential has decreased by about $21 \mathrm{eV}$ from the unperturbed value [24].

Figure 1(a) shows the momentum correlation in the direction parallel to the laser field. The horizontal axis represents the component $k_{a}^{\|}$of one electron, and the vertical axis the component $k_{b}^{\|}$of the other electron. In agreement with earlier measurements $[14,15,28]$, we find a maximum when both electrons are ejected on the same side with similar momentum.

Within the recollision model, the parallel momenta have a simple physical interpretation. The time dependent laser field labels the time of ejection of the electrons. Thus, we gain information about the time the two electrons are reemitted.

Let us assume that (a) the reemitted electrons leave the atom with no significant energy and (b) electron-electron

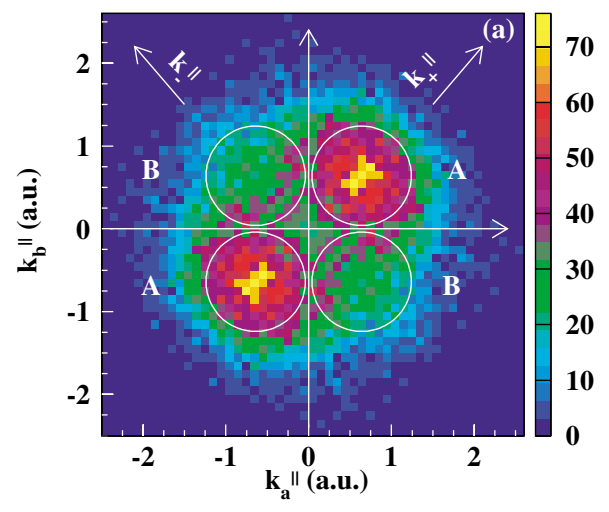

$\begin{array}{llllllllllllllll}0 & 10 & 20 & 30 & 40 & 50 & 60 & 70 & 0 & 5 & 10 & 15 & 20 & 25 & 30 & 35\end{array}$

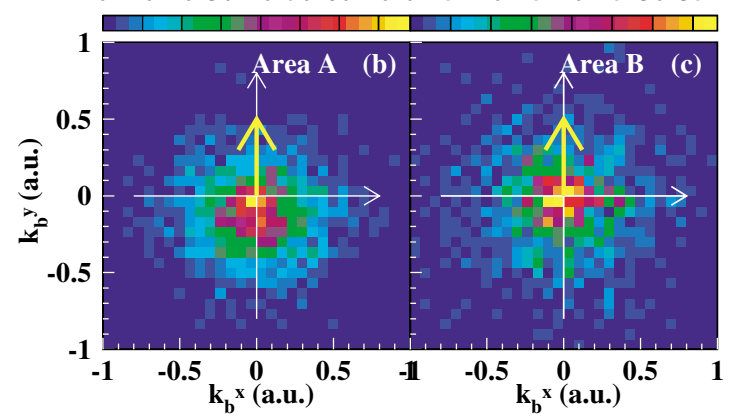

FIG. 1 (color online). Double ionization of neon at $1.9 \times$ $10^{14} \mathrm{~W} / \mathrm{cm}^{2}, 800 \mathrm{~nm}, 40$ fsec. (a) Horizontal axis: momentum of electron $a$ parallel to the polarization direction. Vertical axis: momentum of electron $b$ parallel to the polarization direction. The areas indicated by the circles show the region of events selected in (b) and (c). (b) Momentum components of electron $b$ in the plane perpendicular to the polarization; the perpendicular momentum of electron $a$ is along the positive $y$ axis as shown by the arrow. Only events within the circles in region A have been selected. (c) Same as (b) but for events in circles in region $\mathrm{B}$.

momentum exchange in the final state is negligible. Based on these assumptions, the parallel momentum $k_{a, b}^{\|}$of each electron results exclusively from the acceleration in the optical field:

$$
k_{a, b}^{\|}=2 \sqrt{U_{p}} \sin \omega t_{\text {ion }} .
$$

Thus, for events with $k_{a}^{\|} \cong k_{b}^{\|}$along the diagonal $k_{+}^{\|}=$ $k_{a}^{\|}+k_{b}^{\|}$, both electrons were ejected simultaneously (or any other time when $\sin \omega t_{\text {ion }}$ has the same value). The momentum difference $k_{a}^{\|}-k_{b}^{\|}$measures the time difference between the reemission of the two electrons.

Events in quadrants two and four (region B) are consistent with electrons that leave the ion at a significantly different time [15]. If this is true, there can be no final state interaction. Figure 1(c) shows the momentum component of one electron in the plane perpendicular to the polarization for events indicated in region B in Fig. 1(a). The transverse momentum of the other electron defines the positive $y$ axis of this graph as indicated by an arrow, whose length does not imply a restriction to its absolute value. The momentum distribution in Fig. 1(c) is 
approximately Gaussian with a $1 / e$ width of 0.3 a.u. in both directions.

The $1 / e$ width of the lateral momentum distribution is very close to what we measure for tunnel ionization in the same field [23]. The lateral electron momentum distribution is given by the spatial width of the barrier [Eq. (1)] and is the minimum allowed by the uncertainty principle. Thus, the electrons do not retain any angular momenta that they might have had before escaping, and we might also expect their longitudinal excess momenta to be small [assumption (a)].

Our observations of a loss of repulsion in region B supports an argument by Feuerstein et al. [15] that these events are created by recollision with electron impact excitation followed by a time-delayed field ionization of the excited state. In contrast, final state interaction is evident for electrons in region A. Figure 1(b) shows that the momentum distribution of the second electron is biased in the opposite direction to the first electron. The offset is 0.1 a.u., but the width of the distribution is approximately the same as in Fig. 1(c). This result is consistent with electron repulsion in the final state [16], when electrons are emitted almost simultaneously.

Although we measure evidence that the electrons interact in their final state, the momentum transferred by the interaction is too small to make Eq. (2) invalid. Using Eq. (2), $k_{a}^{\|}=k_{b}^{\|}=0.65$ a.u. implies a reemission phase $\omega t_{\text {ion }}$ of $30^{\circ}$ off the field maximum. The observed repulsion shows that the electrons must be reemitted within a relatively small time window within the same field quad-

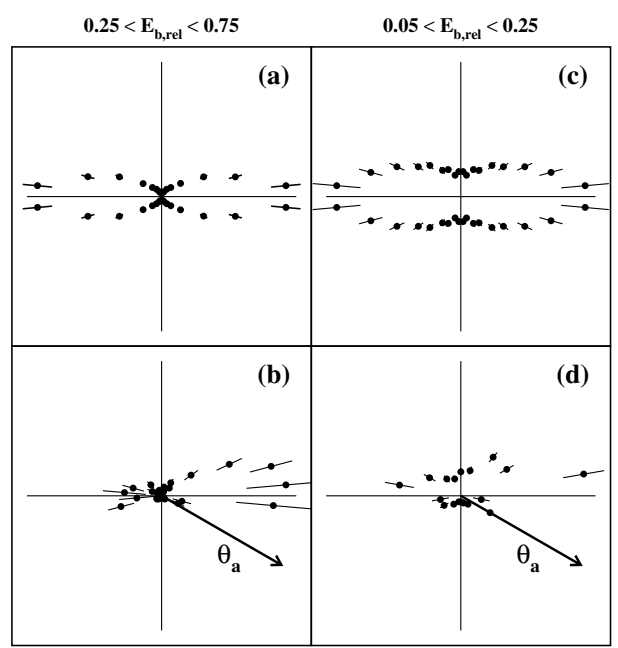

FIG. 2. Angular distribution of electron $b$. The polarization axis is horizontal, $10<\left(E_{a}+E_{b}\right)<24 \mathrm{eV}$. (a) All electrons with an energy sharing $0.25<E_{b \text {,rel }}<0.75$ (see text). Integrated over all angles and energies of electron $a$, (b) as (a) but for a fixed polar angle $\theta_{a}=30^{\circ} \pm 10^{\circ}$ of electron $a$. Both electrons are coplanar [azimuthal angle $\left(\phi_{a}-\phi_{b}\right)<$ $0^{\circ} \pm 40^{\circ}$ lower half and $\left(\phi_{a}-\phi_{b}\right)<180^{\circ} \pm 40^{\circ}$ upper half]. Shown is the differential rate $d \sigma^{4} / d E_{1} d E_{2} d \Omega_{a} d \Omega_{b}$. (c),(d) Corresponding data to (a) and (b) but for an energy sharing of $0.05<E_{b \text {,rel }}<0.25$. rant. This removes some aspects of the $\sin \omega t_{\text {ion }}$ ambiguity in Eq. (2).

The observation for region $\mathrm{A}$ is consistent with the electrons being stored in double excited states between the time of recollision and the time the laser field increases sufficiently for them to escape over the barrier. The essential role of double excited states in threshold double ionization has been previously proposed [24,25].

We now turn to the correlated angular distributions of the two electrons. Figures 2(b), 2(d), and 3 show fully differential cross sections; i.e., they are not integrated over any of the observables. The sum energy of both electrons has been constrained to $10<\left(E_{a}+E_{b}\right)<$ $24 \mathrm{eV}$ and the polar emission angle $\theta_{a}$ of one electron (indicated by the arrow in the figures) with respect to the polarization. In addition, the figures show the coplanar geometry. That is, the azimuthal angles $\phi_{a, b}$ are fixed to confine both electrons to the plane of the figure. Figures 2(a) and 2(c) show the angular distribution of electrons with different energy sharing, $E_{b, \text { rel }}=E_{b} /$ $\left(E_{a}+E_{b}\right)$. The electrons are driven by the field into narrow cones along the polarization axis. For the asymmetric energy sharing, some flux is also seen in the perpendicular direction, while for the equal energy sharing there is almost a node at $\theta_{b}=90^{\circ}$ (compare [13]). If now the direction and energy of the second electron is fixed [Fig. 2(b)], we observe a double lobe structure with significantly more intensity for both electrons being ejected to the same side than to opposite sides. The main lobe is bent upward and clearly shows the influence of electron repulsion. The smaller lobe to the left side is almost symmetric. This is in accordance with our preceding interpretation that the emitted electrons are time delayed, and hence these electrons do not see the repulsion. For unequal energy sharing [Fig. 2(d)], the asymmetry between the two lobes is reduced, and a significant

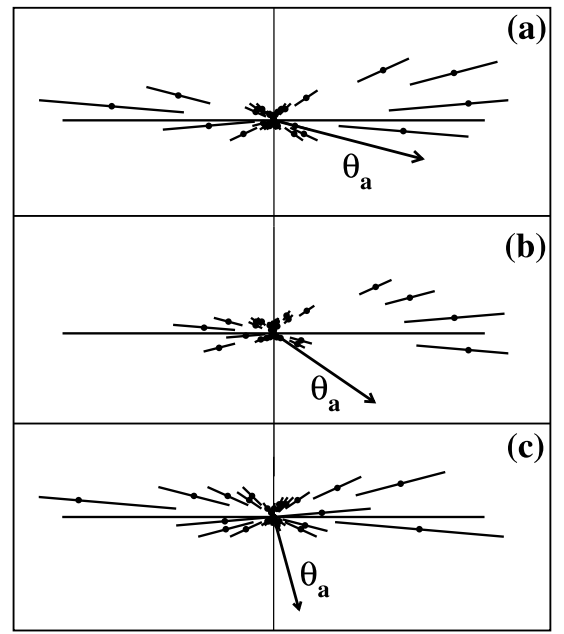

FIG. 3. As Fig. 2(b), but for equal energy sharing $0.25<$ $E_{b, \text { rel }}<0.75$, and (a) $\theta_{a}=15^{\circ} \pm 5^{\circ}$, (b) $\theta_{a}=35^{\circ} \pm 15^{\circ}$, (c) $\theta_{a}=75^{\circ} \pm 15^{\circ}$. 
flux not only in the narrow cone along the polarization axis but also perpendicular to it is seen.

Figure 3 shows the dependence of the angular distribution on the angle of the first electron for equal energy sharing. For all angles $\theta_{a}$, we observe a very narrowly directed emission of the other electron close to the polarization axis accompanied by a downward shift due to electron repulsion. The left-right symmetry is gradually restored when going from $\theta_{a}=30^{\circ}$ to $90^{\circ}$.

In conclusion, unlike the case of single photon double ionization [3] where electron repulsion leads to a preferred back-to-back emission, in strong field double ionization both electrons tend to be observed in the same direction. This is because of the commanding role of the laser field along the field direction. In the perpendicular direction, the field plays a smaller role. Back-to-back emission occurs in the direction perpendicular to the laser field.

The low lateral momentum of the electron implies that the longitudinal momentum when they are reemitted from the atom is also low. Therefore, Eq. (2) is applicable to both electrons independently, and so the time difference between the emission of the two electrons is resolvable up to the multivalued nature of $\sin \omega t_{\text {ion }}$ by measuring the longitudinal momentum of both electrons. Since the lateral momentum can be used to remove the $\sin \omega t_{\text {ion }}$ ambiguity, the path is now clear for resolving the dynamics of threshold double ionization with much better than 1/4 laser cycle (attosecond) precision.

We are indebted to Horst Schmidt-Böcking and Achim Czasch for indispensable support in this project and to Andreas Becker and Robert Moshammer for valuable discussions. The experimental work is supported by the NRC HGF science \& technology fund, DFG, and BMBF (Internationales Büro). A.S. thanks the Studienstiftung des deutschen Volkes for support. Th.W. thanks Graduiertenförderung des Landes Hessen and the Alexander von Humboldt Stiftung for financial support.

*Email: Doerner@ikf.uni-frankfurt.de

[1] A. Huetz and J. Mazeau, Phys. Rev. Lett. 85, 530 (2000).

[2] A. Knapp, A. Kheifets, I. Bray, Th. Weber, A. L. Landers, S. Schössler, T. Jahnke, J. Nickles, S. Kammer, O. Jagutzki, L. Ph. Schmidt, T. Osipov, J. Rösch, M. H. Prior, H. Schmidt-Böcking, C. L. Cocke, and R. Dörner, Phys. Rev. Lett. 89, 033004 (2002).

[3] J. Briggs and V. Schmidt, J. Phys. B 33, R1 (2000).

[4] A. Kheifets and I. Bray, J. Phys. B 31, L447 (1998).

[5] M. Pont and R. Shakeshaft, Phys. Rev. A 51, R2676 (1995).

[6] L. Malegat, P. Selles, and A. K. Kazansky, Phys. Rev. Lett. 85, 4450 (2000).

[7] J. Colgan and M. S. Pindzola, Phys. Rev. A 65, 032729 (2002).
[8] B. Walker, B. Sheehy, L. F. DiMauro, P. Agostini, K. H. Schafer, and K. C. Kulander, Phys. Rev. Lett. 73, 1227 (1994).

[9] Th. Weber, M. Weckenbrock, A. Staudte, L. Spielberger, O. Jagutzki, V. Mergel, G. Urbasch, M. Vollmer, H. Giessen, and R. Dörner, Phys. Rev. Lett. 84, 443 (2000); J. Phys. B 33, L127 (2000).

[10] R. Moshammer, B. Feuerstein, W. Schmitt, A. Dorn, C. D. Schröter, J. Ullrich, H. Rottke, C. Trump, M. Wittmann, G. Korn, K. Hoffmann, and W. Sandner, Phys. Rev. Lett. 84, 447 (2000).

[11] R. Lafon, J. L. Chaloupka, B. Sheehy, P. M. Paul, P. Agostini, K. C. Kulander, and L. F. DiMauro, Phys. Rev. Lett. 86, 2762 (2001).

[12] E. R. Peterson and P. H. Bucksbaum, Phys. Rev. A 64, 053405 (2001).

[13] R. Moshammer, J. Ullrich, B. Feuerstein, D. Fischer, A. Dorn, C. D. Schröter, J. R. C. Lopez-Urrutia, C. Höhr, H. Rottke, C. Trump, M. Wittmann, G. Korn, K. Hoffmann, and W. Sandner, J. Phys. B 36, L113 (2003).

[14] Th. Weber, H. Giessen, M. Weckenbrock, G. Urbasch, A. Staudte, L. Spielberger, O. Jagutzki, V. Mergel, M. Vollmer, and R. Dörner, Nature (London) 405, 658 (2000).

[15] B. Feuerstein, R. Moshammer, D. Fischer, A. Dorn, C. D. Schröter, J. Deipenwisch, J. R. C. Lopez-Urrutia, C. Höhr, P. Neumayer, J. Ullrich, H. Rottke, C. Trump, M. Wittmann, G. Korn, and W. Sandner, Phys. Rev. Lett. 87, 043003 (2001).

[16] M. Weckenbrock, A. Becker, A. Staudte, S. Kammer, M. Smolarski, V. R. Bhardwaj, D. M. Rayner, D. M. Villeneuve, P. B. Corkum, and R. Dörner, Phys. Rev. Lett. 91, 123004 (2003).

[17] R. Dörner, Th. Weber, M. Weckenbrock, A. Staudte, M. Hattass, H. Schmidt-Böcking, R. Moshammer, and J. Ullrich, Advances in Atomic Molecular and Optical Physics, edited by B. Bederson and H. Walther (Academic Press, New York, 2002), p. 48.

[18] G. L. Kamta and A. F. Starace, Phys. Rev. Lett. 86, 5687 (2001).

[19] A. Becker and F. H. M. Faisal, Phys. Rev. A 50, 3256 (1994).

[20] M. Yu. Kuchiev, JETP Lett. 45, 404 (1987).

[21] P. B. Corkum, Phys. Rev. Lett. 71, 1994 (1993).

[22] A. Jaron and A. Becker, Phys. Rev. A 67, 035401 (2003).

[23] H. Niikura, F. Legare, R. Hasbani, M. Yu. Ivanov, D. M. Villeneuve, and P. B. Corkum, Nature (London) 417, 917 (2002).

[24] T. Brabec, M. Yu. Ivanov, and P. B. Corkum, Phys. Rev. A 54, R2551 (1996).

[25] R. Panfili, S. L. Haan, and J. H. Eberly, Phys. Rev. Lett. 89, 113001 (2002).

[26] J. Ullrich, R. Moshammer, A. Dorn, R. Dörner, L. Ph. Schmidt, and H. Schmidt-Böcking, Rep. Prog. Phys. 66, 1463 (2003).

[27] M. Lewenstein, Ph. Balcou, M. Y. Ivanov, A. L'Huiller, and P. B. Corkum. Phys. Rev. A 49, 2117 (1994).

[28] E. Eremina, X. Liu, H. Rottke, W. Sandner, A. Dreischuh, F. Lindner, F. Grasbon, G. G. Paulus, H. Walther, R. Moshammer, B. Feuerstein, and J. Ullrich, J. Phys. B 36, 3269 (2003). 